\title{
Application of time domain two - dimensional acquisition method in spread
}

\section{spectrum system}

\author{
Xu Xingyuan, Xia Lili, Liu Sifang \\ China Satellite Maritime Tracking and Control Department, Jiangyin, 214431, China
}

Key words: two-dimensional acquisition; spread spectrum; frequency estimation

\begin{abstract}
In this paper, the principle of time-domain two-dimensional acquisition method is described, and the effects of symbol offset and Doppler frequency estimation error on the correlation peak are analyzed in detail, so as to determine the size of pseudo-code phase and Doppler frequency step. It is of reference value for signal acquisition of spread spectrum system.
\end{abstract}

\section{Introduction}

Fast and reliable acquisition target is the most important problem in the system of space spread spectrum measurement and control system. There are two main factors that affect the normal operation of the system: code phase and carrier frequency uncertainty. The resolution of the code phase is less than 1chip. The resolution of the carrier center frequency allows the despread signal to fall within the frequency band of the relevant filter and the local carrier frequency always tracks the frequency of the input signal carrier so that the despread signal is always fall within the relevant filter frequency band, to ensure that the demodulator can work properly.

\section{Capture principle}

Time domain two-dimensional capture method is one of the most basic pseudo-code capture method, the use of this method, the specific capture process is as follows:

A). First set the local oscillator (by the NCO implementation) for a frequency value, and the received input signal mixing, the signal changes to the baseband;

B). The baseband signal and the local pseudo-code sequence together into the correlator to multiply and accumulate;

C). Compared with the decision threshold, if the threshold is exceeded, the capture is considered successful and the system goes into the tracking state. Traversing all the phases of a pseudocode period, the searching process of the code phase direction ends at the frequency point;

D). If the search of the code phase direction at this frequency does not make the output of the correlator exceed the decision threshold, the local oscillation frequency slips one step to the next frequency point, repeats the above process and performs a new round of searching process until Traverse all the Doppler frequencies.

Time domain two-dimensional capture method is a serial search method, not only to search for the code phase, but also to search the carrier Doppler shift, the search schematic diagram shown in Figure 1.

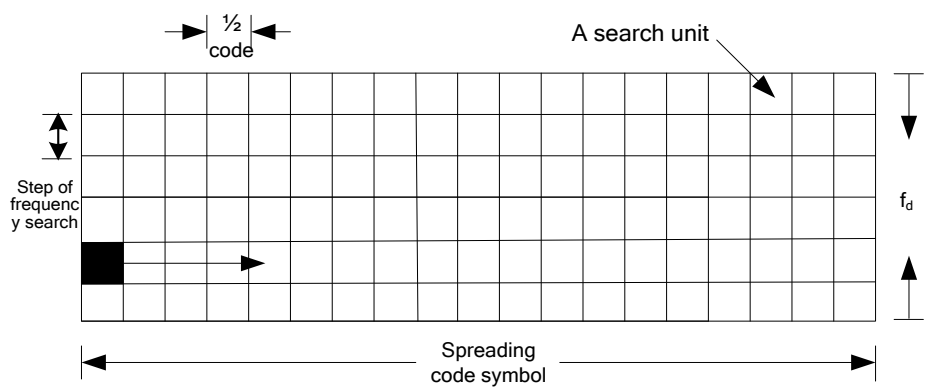

Figure 1. Time domain two-dimensional serial search diagram 
Figure 2 shows the time domain two-dimensional capture algorithm simulation diagram, simulation conditions:
A). Pseudo-random code selection code length of 1023 Gold code sequence, pseudo-code rate 5.115Mchip / s;
B). The accumulation time is $0.2 \mathrm{~ms}$;
C). Phase of code search step is set to half a code; Doppler frequency search step is $2 \mathrm{kHz}$;
D. The Doppler shift is $50 \mathrm{kHz}$ and the code phase offset is 320 chips.

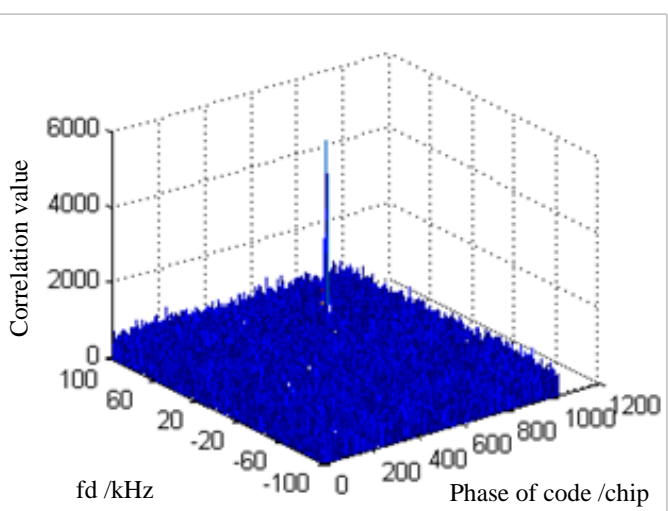

Figure 2. Time domain two - dimensional capture results simulation diagram

Figure 2 shows the simulation results of two-dimensional acquisition in time domain. According to Fig. 2, a significant correlation peak can occur at a position where the code phase shift is 320 chips.

\section{The effect of symbol offset and Doppler frequency estimation error}

In the time-domain two-dimensional acquisition method, the searching step of the symbol and Doppler frequency directly affects the acquisition performance and the search time. For signals with low signal-to-noise ratio (SNR), when the search step is too large, the peak value may not be detected and the probability of false alarm will be increased. If the search step is too small, it will increase the detection time and reduce the capture speed. Select the search step size for the symbol and carrier Doppler. The effects of the symbol offset and the Doppler frequency estimation error on the correlation peak are quantitatively analyzed.

Assume that the input IF signal is:

$$
r(t)=A d(t) P N(t-\varepsilon \tau) \cos \left(\left(\omega_{0}+\omega_{d}\right) t+\varphi_{0}\right)+N(t)
$$

Regardless of the noise effect, the correlator output is:

$$
I=\frac{A T}{2} \cdot(1-\varepsilon) \cdot s a \frac{\Delta \omega T}{2} \cdot \frac{s a\left[\frac{\Delta \omega(1-\varepsilon) \tau}{2}\right]}{s a \frac{\Delta \omega \tau}{2}} \cos \left[\frac{\Delta \omega T}{2}+\frac{\Delta \omega \varepsilon \tau}{2}+\Delta \phi\right]
$$

Similarly available:

$$
Q=\frac{A T}{2} \cdot(1-\varepsilon) \cdot \operatorname{sa} \frac{\Delta \omega T}{2} \cdot \frac{\operatorname{sa}\left[\frac{\Delta \omega(1-\varepsilon) \tau}{2}\right]}{\operatorname{sa} \frac{\Delta \omega \tau}{2}} \sin \left[\frac{\Delta \omega T}{2}+\frac{\Delta \omega \varepsilon \tau}{2}+\Delta \phi\right]
$$

The decision variables are:

$$
y=\sqrt{I^{2}+Q^{2}}=\frac{A T}{2}\left|(1-\varepsilon) \cdot s a \frac{\Delta \omega T}{2} \cdot \frac{s a\left[\frac{\Delta \omega(1-\varepsilon) \tau}{2}\right]}{s a \frac{\Delta \omega \tau}{2}}\right|
$$

Therefore, the signal correlation amplitude is reduced by the pseudocode phase offset and the Doppler frequency 
estimation error to be several times that of the pseudo-code synchronization.

When the integration time T is $1 \mathrm{~ms}$, the PN code length is 1023 , the pseudo code symbol width is $1 / 1023 \mathrm{~ms}$, the pseudo code phase offset range [0,1] chip, the Doppler frequency estimation error $[-10 \mathrm{kHz}, 10 \mathrm{kHz}] \mathrm{The}$ influence of the pseudo-code phase offset and Doppler frequency estimation error on the correlation peak is shown in Fig.

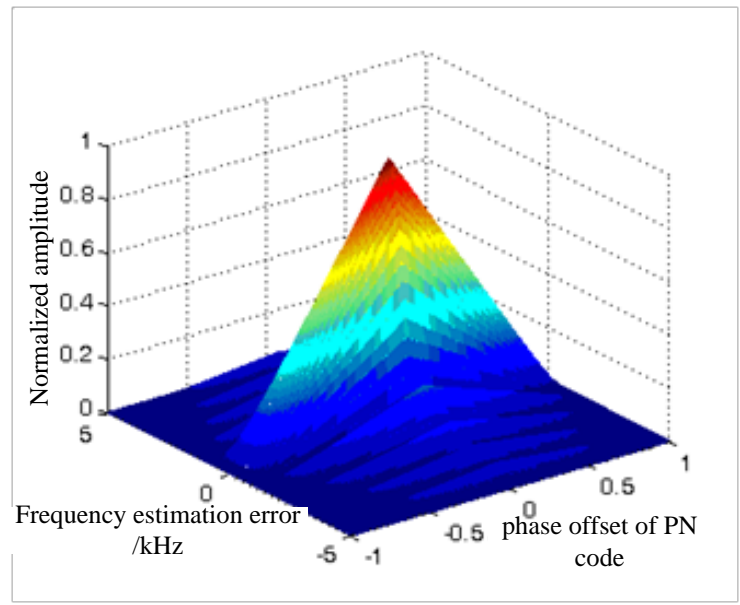

Figure 3. Influence of Pseudo Code Phase Offset and Doppler Frequency Estimation Error on Correlation Peak In the following, the performance of correlation peak is analyzed for the pseudo-code phase estimation error and Doppler frequency estimation error, respectively.

A). When the Doppler frequency estimation error is 0, the influence of pseudocode on the correlation peak is:

$$
y=\sqrt{I^{2}+Q^{2}}=\frac{A T}{2}|1-\varepsilon|
$$

It can be seen that the influence of the pseudo-code phase shift on the correlation peak is linear, which is $|1-\varepsilon|$ times that of the complete synchronization. As shown in Figure 4.

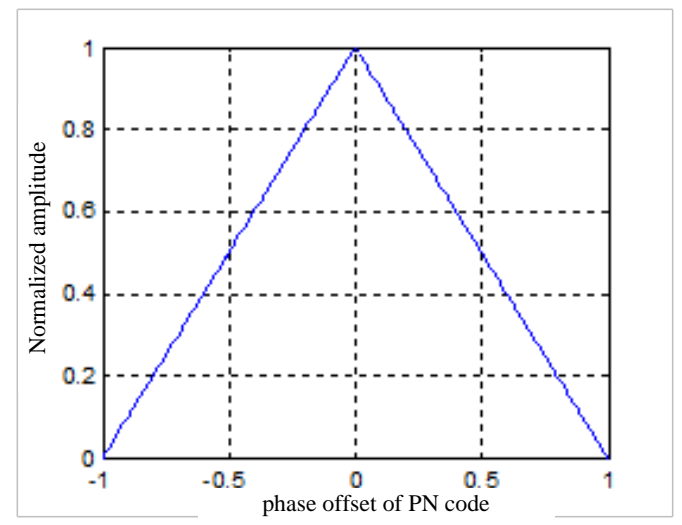

Figure 4. Influence of Pseudocode Phase Offset on Correlation Peak

It can be seen from Fig.4 that when the absolute value of the PN code phase estimation error is smaller, the correlation peak is bigger; when the error exceeds one symbol width, there is no correlation peak output, the system can not capture the target.

B). When the pseudo-code phase is completely aligned, when A = B, the Doppler frequency estimation error affects the correlation peak:

$$
y=\sqrt{I^{2}+Q^{2}}=\frac{A T}{2}\left|s a \frac{\Delta \omega T}{2}\right|
$$


It can be seen that the loss of the signal energy is $s a^{2}(\Delta \omega T$ / 2) times because the Doppler shift estimation is not accurate and the signal correlation amplitude is reduced to $|s a(\Delta \omega T / 2)|$ times the Doppler frequency shift estimate is completely correct. According to the characteristics of the Sa function, its $0.9 \mathrm{~dB}$ point is about 0.25 times the mainlobe bandwidth. Therefore, it is necessary to keep the frequency search stepping within $0.25 / \mathrm{T}$ to ensure that the correlation loss caused by Doppler uncertainty is less than $0.9 \mathrm{~dB}$. When the signal to noise ratio is very low, you can reduce the frequency search step by appropriate to reduce the attenuation of the relevant amplitude.

The three curves in Figure 5 show the influence of the Doppler shift estimation error on the correlation peak when the integration times are $0.2 \mathrm{~ms}, 0.4 \mathrm{~ms}$ and $0.6 \mathrm{~ms}$, respectively.

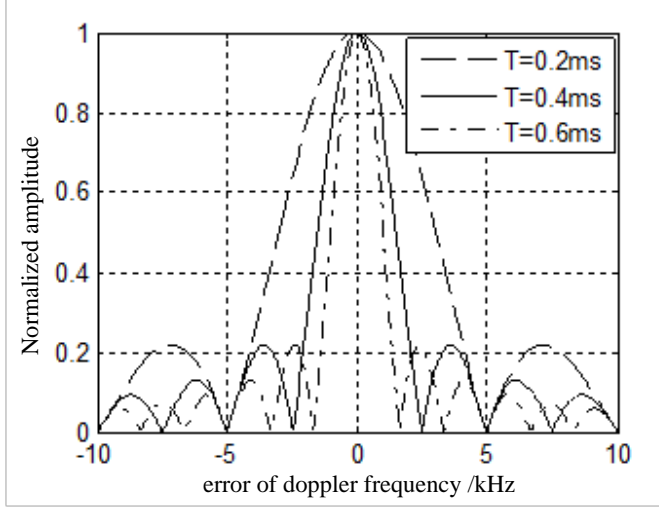

Figure 5. Influence of Doppler Frequency Estimation Error on Correlation Peak

It can be seen from Figure 5, when the single accumulation time is $0.2 \mathrm{~ms}$, the peak of the first mainlobe of the cutoff frequency of $5 \mathrm{kHz}$; when the cumulative time of $0.4 \mathrm{~ms}$, the cut-off frequency of $2.5 \mathrm{kHz}$; when the cumulative time of $0.6 \mathrm{~ms}$, The maximum Doppler estimation error that can tolerate is only $1.67 \mathrm{kHz}$. Obviously, the longer the integration time, the narrower the width of the main lobe and the steeper the correlation peak output, the more sensitive to the Doppler estimation error; therefore, the maximum step size of the frequency search will be reduced. While the decrease of the frequency search step leads to the decrease of the acquisition speed. Generally, each frequency search step is controlled to 0.25 / T to ensure that the associated loss caused by Doppler uncertainty is less than $0.9 \mathrm{~dB}$. When the signal to noise ratio is very low, you can reduce the frequency search step by appropriate to reduce the attenuation of the relevant amplitude.

\section{Summary}

In this paper, the principle of time-domain two-dimensional acquisition algorithm is analyzed, and the influence of symbol offset and Doppler frequency estimation error on the correlation peak is deduced, and the searching step of pseudocode and Doppler frequency is determined. It is of reference value for signal acquisition of spread spectrum system.

\section{Reference}

[1] J.K Holmes, C Chen. Acquisition time performance of PN spread-spectrum systems[J]. IEEE Transactions on communications,2011:778-784

[2] Van Dierendonck. GPS Receivers in Global Positioning System [J].Theory and Application.Vol.I.2006. 329 407.

[3] Grant P.M, Spangenberg S.M, Scott J.etal. Doppler estimation for fast acquisition in spread spectrum communication systems [J]. IEEE 2008

[4] J. C. Lin. No coherent SPRT Based Acquisition Scheme for DSSS. IEEE Electronics Letters, 2012, 34(6):13 25 
[5] C. Baum, V Veeravalli. Hybrid acquisition schemes for direct sequence CDMA systems in: Conference Record, IEEE International Communications Conference on Communications, 2012, 3:1433 1437 